\title{
Some difficulties in the theory of diffusion-controlled growth in substitutionally alloyed steels
}

\author{
H. K. D. H. Bhadeshia ${ }^{1, *}$ \\ University of Cambridge \\ Materials Science and Metallurgy \\ Pembroke Street, Cambridge CB2 3QZ, U. K. \\ www.msm.cam.ac.uk/phase-trans
}

\begin{abstract}
The theory for the diffusion-controlled growth of ferrite in steels that also contain substitutional solutes is fraught with difficulties when it comes to transformation at large supersaturations, where the bulk compositions of the ferrite and austenite do not differ much, but where local-equilibrium is nevertheless maintained at the transformation front. This requires the existence of a narrow variation in substitutional solute content in the austenite at the interface (so-called 'concentration spike') - so narrow that it has no physical meaning. Drawing on the theory for spinodal reactions, it is demonstrated here that there is a substantial penalty associated with the creation of such sharp changes in composition. Therefore, the spikes would never occur in practice. The actual distribution of solute would be over distances orders of magnitude larger than currently calculated, leading to slower growth rates than are predicted currently. The consequences of this conclusion place doubt both on the transition from local to paraequilibrium, and whether the latter state exists at all for reconstructive transformations.
\end{abstract}

Keywords: Steels, diffusion-controlled growth, phase transformations, local-equilibrium, paraequilibrium, reconstructive diffusion

\section{Introduction}

It could be argued quite reasonably that the interpretation of growth rate data on the formation of ferrite ${ }^{2}$ in steels, is in a state of crisis. This is because of a failure to account for the limitations of experimental techniques and genuine difficulties with the theory of diffusion-controlled growth in multicomponent steels. One might be forgiven in deducing from the published literature

\footnotetext{
*Corresponding author

${ }^{1}$ hkdb@cam.ac.uk

${ }^{2}$ Strictly allotriomorphic ferrite
} 
that whenever there is a gap between theory and experiment, it is explained by appealing to free energy dissipations that have little in the way of supporting evidence. As pointed out in recent reviews [1, 2], this necessitates the choice of fitting parameters including interface thickness, interface diffusivity, binding energies etc. which make the theory less useful as a predictive tool. In contemporary discussion, however, a key factor relevant to the theory is neglected, i.e. that difficulties arise when sharp concentration profiles are developed, the solution to which may give a more physically realistic picture of allotriomorphic ferrite growth. This is the focus of the present contribution.

To explain the issues properly, it is necessary to first introduce, in some detail, the basic concepts of diffusion-controlled growth in $\mathrm{Fe}-\mathrm{C}$ and in alloyed steels where the diffusivity of the substitutional solutes can be many orders of magnitude smaller than of the interstitial carbon. How then is it possible to maintain local equilibrium for both kinds of solutes at the moving interface?

\section{Diffusion-controlled growth in binary system}

There are many processes that participate in the events leading to the transformation of austenite $(\gamma)$ into ferrite $(\alpha)$ by the translation of the $\alpha / \gamma$ interface [3-5]. These include the diffusion of solute ahead of the transformation front, the transfer of atoms across the interface and the movement of solute atoms that might be segregated to the interface. All of these in principle dissipate the free energy that is available for transformation, but the process that accounts for the majority of the dissipation is said to be rate controlling. Diffusion-controlled growth is therefore said to occur when most of the available free energy is used in driving the diffusion of solutes ahead of the interface.

In the case of alloys, two phases are in equilibrium when the chemical potentials $(\mu)$ of the solutes are identical in each phase, Fig. 1a. The chemical potential of a solute when multiplied by its concentration represents the contribution of that solute to the total free energy of the phase. If $\mu_{\mathrm{C}}^{\alpha}$ is identical to $\mu_{\mathrm{C}}^{\gamma}$ then there is no driving force for carbon to diffuse between the phases even if the compositions $c^{\alpha \gamma}$ and $c^{\gamma \alpha}$ are different. ${ }^{3}$ Here $c^{\alpha \gamma}$ is the composition of ferrite that is in equilibrium with austenite, and a similar logic applies to the other term. These compositions are not independent but rather, are tied together. Fig. 1b illustrates a tie-line for temperature $T_{1}$ that connects the equilibrium compositions $c^{\alpha \gamma}$ and $c^{\gamma \alpha}$ of ferrite and austenite respectively. There is a unique tie-line at a given temperature, which passes through the average alloy composition $\bar{c}$.

If a reasonable assumption is now made that for diffusion-controlled growth, the compositions at the flat transformation front are in local equilibrium, then the composition profile that develops during transformation at $T_{1}$ is that illustrated in Fig. 1c, assuming that the far-field value is unaffected and therefore

\footnotetext{
${ }^{3}$ The term $c$ is the concentration per unit volume throughout this paper. On the other hand, $x$ represents an atomic fraction.
} 
fixed at $\bar{c}$. The concentrations $c^{\alpha \gamma}$ and $c^{\gamma \alpha}$ are maintained at the interface throughout the growth process because the chemical potentials of the solute in the ferrite and austenite in contact at the interface are then equal. To achieve this constancy requires that the rate at which solute is partitioned into the austenite as the interface moves is equal to the rate at which it is carried away from the interface by diffusion in the austenite:

$$
\underbrace{\left(c^{\gamma \alpha}-c^{\alpha \gamma}\right) \frac{\partial z^{*}}{\partial t}}_{\text {rate solute partitioned }}=\underbrace{-\left.D \frac{\partial c}{\partial z}\right|_{z=z *}}_{\text {diffusion flux from interface }}
$$

where $t$ is the time set to zero at the beginning of interface motion, $D$ is the solute diffusivity in the parent phase, $z$ is a coordinate normal to the interface with a value $z^{*}$ at the position of the interface. The concentration gradient is evaluated at the position of the interface $\left(z=z^{*}\right)$. The rate of movement of the interface is $v=\partial z^{*} / \partial t$. These concepts can now be generalised to multicomponent alloys.

\section{Diffusion-controlled growth in substitutionally alloyed steel}

Consider now a ternary steel in which one of the solutes is substitutional and the other interstitial, say Fe-Mn-C, with $D_{\mathrm{C}} \gg D_{\mathrm{Mn}}$. The equilibrium conditions are the same as those for a binary alloy, that $\mu_{i}^{\alpha}=\mu_{i}^{\gamma}$ for $i=\mathrm{Mn}, \mathrm{Fe}$ or C. Fig. 2a shows that the free energy curves of the binary system now become free energy surfaces in three dimensions, and it is the contact of a tangent plane that is common to both the austenite and ferrite surfaces that defines the tieline connecting the compositions of the two phases in equilibrium. However, the tie-line illustrated is not unique because the tangent plane can be rocked while remaining in contact with the free energy surfaces. This is why there is an entire two-phase $\alpha+\gamma$ phase field at constant temperature. There is therefore a choice of tie-lines ${ }^{4}$ that would all satisfy local equilibrium at the growth front during interface motion in a ternary system. We will see that this is important in the discussion below.

It would be necessary to satisfy two equations of the form of equation 1 , simultaneously, for each of the solutes ${ }^{5}$ :

$$
\begin{aligned}
\left(c_{\mathrm{C}}^{\gamma \alpha}-c_{\mathrm{C}}^{\alpha \gamma}\right) v & =-D_{\mathrm{C}} \nabla c_{\mathrm{C}} \\
\left(c_{\mathrm{Mn}}^{\gamma \alpha}-c_{\mathrm{Mn}}^{\alpha \gamma}\right) v & =-D_{\mathrm{Mn}} \nabla c_{\mathrm{Mn}}
\end{aligned}
$$

where the subscripts refer to the solutes ( 1 for carbon and 2 for $\mathrm{Mn}$ ). The interface velocity $v$ is the $\partial z^{*} / \partial t$ in equation 2 .

\footnotetext{
${ }^{4}$ The whole of the two phase field is defined by tie lines.

${ }^{5}$ in presenting these equations, we neglect cross-diffusion effects of the type described in $[4,6,7]$. This is because their importance in the difficulties to be described later, in the context of growth at high supersaturations, is not significant [8].
} 


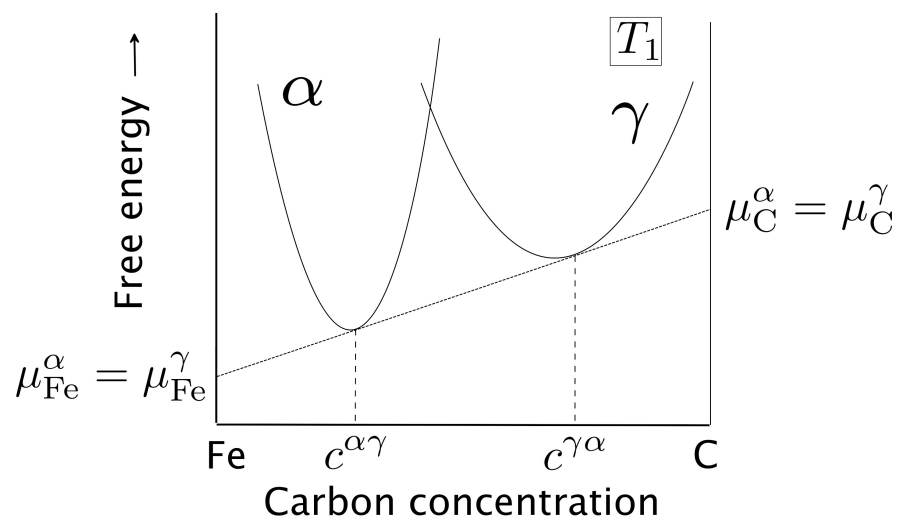

(a)
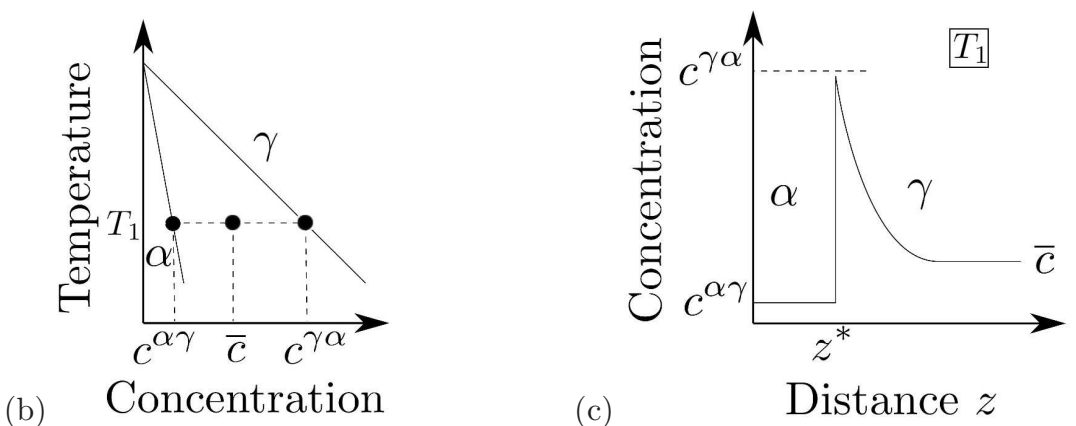

(c)

Distance $z$

Figure 1: (a) Free energy curves for ferrite and austenite for a temperature $T_{1}$, showing how the common tangent defines the compositions that are in equilibrium. (b) Binary phase diagram with tie-line plotted at the transformation temperature $T_{1}$. (c) Concentration profile developed at the $\alpha / \gamma$ interface during diffusion controlled growth. 
Because $D_{\mathrm{C}} \gg D_{\mathrm{Mn}}$, these equations cannot in general be simultaneously satisfied for the tie-line passing through the alloy composition $\bar{c}_{\mathrm{C}}, \bar{c}_{\mathrm{Mn}}$. It is, however, possible to choose other tie-lines which satisfy equation 2 . If the tieline is such that $c_{\mathrm{C}}^{\gamma \alpha}=\bar{c}_{\mathrm{C}}$ (e.g. line $c d$ for alloy $\mathrm{A}$ of Fig. 2a), then $\nabla c_{\mathrm{C}}$ will become very small thus reducing the driving force for carbon diffusion, so that the flux of carbon atoms slows to a rate consistent with that of manganese. Ferrite forming by this mechanism is said to grow by a 'Partitioning, Local Equilibrium' (or P-LE) mechanism, in recognition of the fact that $c_{\mathrm{Mn}}^{\alpha \gamma}$ can differ significantly from $\bar{c}_{\mathrm{Mn}}$, giving considerable partitioning and long-range diffusion of manganese into the austenite.

(a)

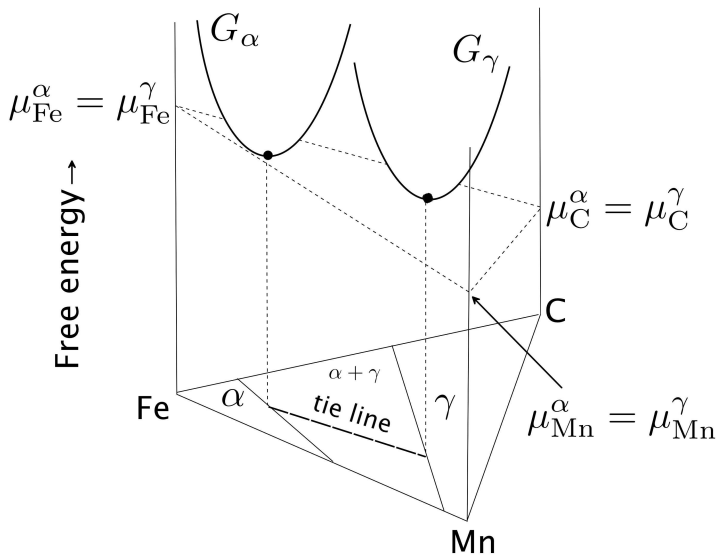

(b)

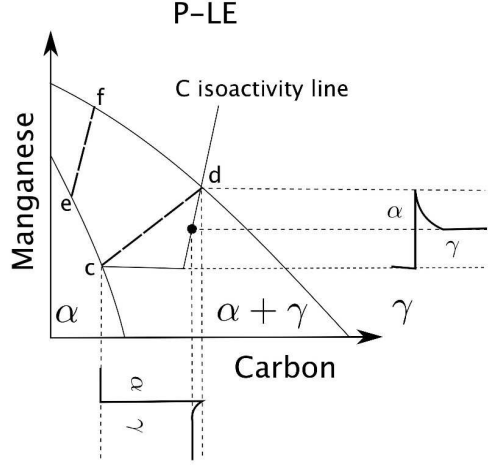

NP-LE

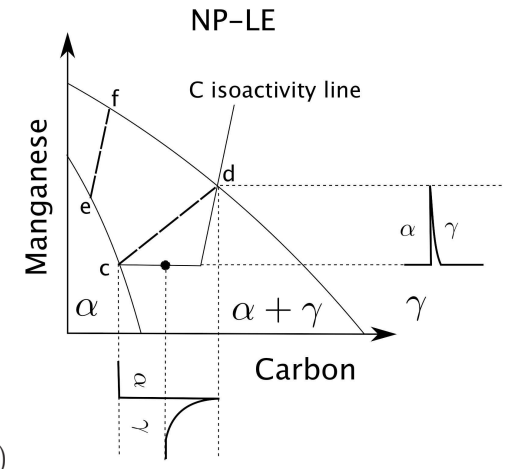

Figure 2: (a) Free energy surfaces of austenite and ferrite, with a common tangent plane defining equilibrium at the compositions given by the contact points of the tangent with the surfaces. The diagram is for a particular temperature. (b) Schematic isothermal section of the Fe-Mn-C system, illustrating ferrite growth occurring with local equilibrium at the $\alpha / \gamma$ interface. Growth at low supersaturations (P-LE) with bulk redistribution of manganese, (c) growth at high supersaturations (NP-LE) with negligible partitioning of manganese during transformation. The bulk alloy compositions are designated by the symbol $\bullet$ in each case. 
An alternative choice of tie-line could allow $c_{\mathrm{Mn}}^{\alpha \gamma} \rightarrow \bar{c}_{\mathrm{Mn}}$ (e.g. line $c d$ for the alloy of Fig. 2b), so that $\nabla c_{\mathrm{Mn}}$ is drastically increased since only very small amounts of $\mathrm{Mn}$ are partitioned into the austenite. The flux of manganese atoms at the interface correspondingly increases and manganese diffusion can then keep pace with that of carbon, satisfying the mass conservation conditions of equation 2. The growth of ferrite in this manner is said to occur by a 'Negligible Partitioning, Local Equilibrium' (or NP-LE) mechanism, in recognition of the fact that the manganese content of the ferrite approximately equals $\bar{c}_{\mathrm{Mn}}$, so that little if any manganese partitions into austenite.

What circumstances determine whether growth follows the P-LE or NP-LE mode? Fig. 3 shows the Fe-Mn-C phase diagram, now divided into domains where either P-LE or NP-LE is possible but not both. The domains are obtained by drawing right-handed triangles on each tie-line in the $\alpha+\gamma$ phase field and joining up all the vertices. If an attempt is made to define NP-LE conditions in the P-LE domain, then the tie-line determining interface compositions will incorrectly show that both austenite and ferrite contain less carbon than $\bar{c}_{\mathrm{C}}$, a circumstance which physically is impossible. An important point to note is that P-LE and NP-LE are modes that operate at low and high supersaturations, respectively. Furthermore, as the composition of the austenite changes, so will the tie-line controlling the interface compositions, but for the purposes of the discussion here, it is assumed that the overlap of diffusion fields (soft-impingement) does not occur.

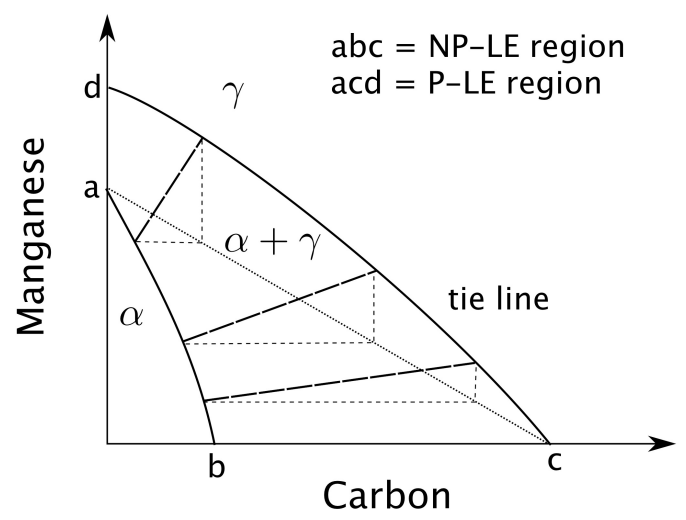

Figure 3: Regions of the two-phase field where either P-LE (low supersaturation) or NP-LE (high supersaturation) modes of transformation are possible.

\section{The problem}

The negligible-partitioning local-equilibrium concepts becomes less realistic as the velocity of the interface increases, i.e. at high supersaturations. This is 
because the extent of the diffusion field for the substitutional solute decreases so much, that it becomes a mathematical formality [3, 6, 9]; indeed, there are no experimental data [reviewed in 2] that confirm the existence of the sharp concentration spikes predicted theoretically in domains where the transformation is supposed to occur by the NP-LE mechanism. It may be argued that an unphysical diffusion profile where the width becomes of the order of an atom size or less would lead essentially to a breakdown of local equilibrium and therefore, give way to paraequilibrium transformation [10-13] in which the Fe/Mn ratio ${ }^{6}$ is maintained constant throughout. We shall return to the issue later in this article.

Coates estimated that for one-dimensional growth with local equilibrium at the interface, the extent of the substitutional-solute diffusion field in the austenite is given approximately by:

$$
z_{\mathrm{Mn}} \approx 2 D_{\mathrm{Mn}} / v
$$

However, Coates appreciated that in these circumstances, where there exist steep concentration gradients, it becomes important to account for the dependence of the diffusion coefficient on the concentration gradient itself, as in the theory of spinodal decomposition. In the latter case, a homogeneous solution can spontaneously develop composition waves that grow in amplitude. However, the wavelengths that develop are not small because there is a cost in creating large concentration gradients. We now consider this scenario in the context of NP-LE growth, but drawing directly on the theory of heterogeneous solutions from spinodal decomposition [14-19]. We shall see that there are factors which oppose the development of sharp concentration spikes, whether they occur in spinodal decomposition or with diffusion-controlled growth. The subject has been reviewed nicely by Hilliard, whose treatment we follow [20].

The free energy of a heterogeneous solution can be expressed by a multivariable Taylor expansion [e.g. 19]:

$$
\begin{aligned}
g\{a, b, \ldots\}= & g\{\bar{c}\}+a \frac{\partial g}{\partial a}+b \frac{\partial g}{\partial b}+\ldots \\
& +\frac{1}{2}\left[a^{2} \frac{\partial^{2} g}{\partial a^{2}}+b^{2} \frac{\partial^{2} g}{\partial b^{2}}+2 a b \frac{\partial^{2} g}{\partial a \partial b}+\ldots\right]+\ldots
\end{aligned}
$$

in which the variables, $a, b, \ldots$ in our context are the spatial composition derivatives $\left(d c / d z, d^{2} c / d z^{2}\right.$, etc). For the free energy of a small volume element containing a one-dimensional composition variation (and neglecting third and high-order terms), this gives

$$
g=g\{\bar{c}\}+\kappa_{1} \frac{d c}{d z}+\kappa_{2} \frac{d^{2} c}{d z^{2}}+\kappa_{3}\left(\frac{d c}{d z}\right)^{2}
$$

${ }^{6} \mathrm{Fe} /$ substitutional-solute ratio. 
where $\bar{c}$ is the average composition and

$$
\begin{aligned}
\kappa_{1} & =\frac{\partial g}{\partial(d c / d z)} \\
\kappa_{2} & =\frac{\partial g}{\partial\left(d^{2} c / d z^{2}\right)} \\
\kappa_{3} & =\frac{1}{2} \frac{\partial^{2} g}{\partial(d c / d z)^{2}} .
\end{aligned}
$$

In this, $\kappa_{1}$ is zero for a centrosymmetric crystal since the free energy must be invariant to a change in the sign of the coordinate $z$.

The total free energy per atom, $g_{i h}$ for the inhomogeneous solution, is obtained by integrating over the volume $V$ :

$$
g_{i h}=\int_{V}\left[g\{\bar{c}\}+\kappa_{2} \frac{d^{2} c}{d z^{2}}+\kappa_{3}\left(\frac{d c}{d z}\right)^{2}\right]
$$

On integrating the third term in this equation by parts:

$$
\int \kappa_{2} \frac{d^{2} c}{d z^{2}}=\kappa_{2} \frac{d c}{d z}-\int \frac{d \kappa_{2}}{d c}\left(\frac{d c}{d z}\right)^{2} d z
$$

As before, the first term on the right is zero, so that an equation of the form below is obtained for the free energy per atom of a heterogeneous system:

$$
g_{i h}=\int\left[g\{\bar{c}\}+v_{\mathrm{a}}^{3} \kappa(\nabla c)^{2}\right] d V
$$

where $v_{\mathrm{a}}$ is the volume per atom and $\kappa$ is known as the gradient energy coefficient. The term $g\{\bar{c}\}$ is the free energy of a homogeneous solution with the average concentration $\bar{c}$. The interpretation of this equation is that gradients of concentration lead to an increase in the free energy, so that the formation of steep gradients will in general be opposed. Before discussing the implications of this, it is necessary to consider an additional term which arises because the lattice parameter varies with concentration. The resulting elastic strains add to the free energy. If the dimensionless quantity $\eta$ is defined as $\mathrm{d} \ln a / \mathrm{d} x$, where $a$ is the lattice parameter and $x$ the atomic fraction of concentration, then the strain energy per atom has two components. The first is approximately $\eta^{2} v_{\mathrm{a}}^{3} E(c-\bar{c})^{2} /(1-\nu)$ where $E$ is the Young's modulus and $\nu$ is the Poisson's ratio, and the second contribution has a dependence on $(\nabla c)^{2}$ and hence can be incorporated into the gradient energy coefficient [20,21].

The strain energy term turns out to be rather small so we focus instead on the gradient energy component. Fig. 4 shows the increase in free energy of the solution as a consequence of the substitutional solute concentration spike in the austenite at the $\gamma / \alpha$ interface during negligible partitioning local equilibrium growth. The diffusion distance is intended to represent the width of the spike. The calculations assume a gradient energy coefficient of $3.85 \times 10^{-10} \mathrm{~J} \mathrm{~m}^{-1}$ based on the Fe-Cr system $[22,23]$ and that $(x-\bar{x})=0.03$. 


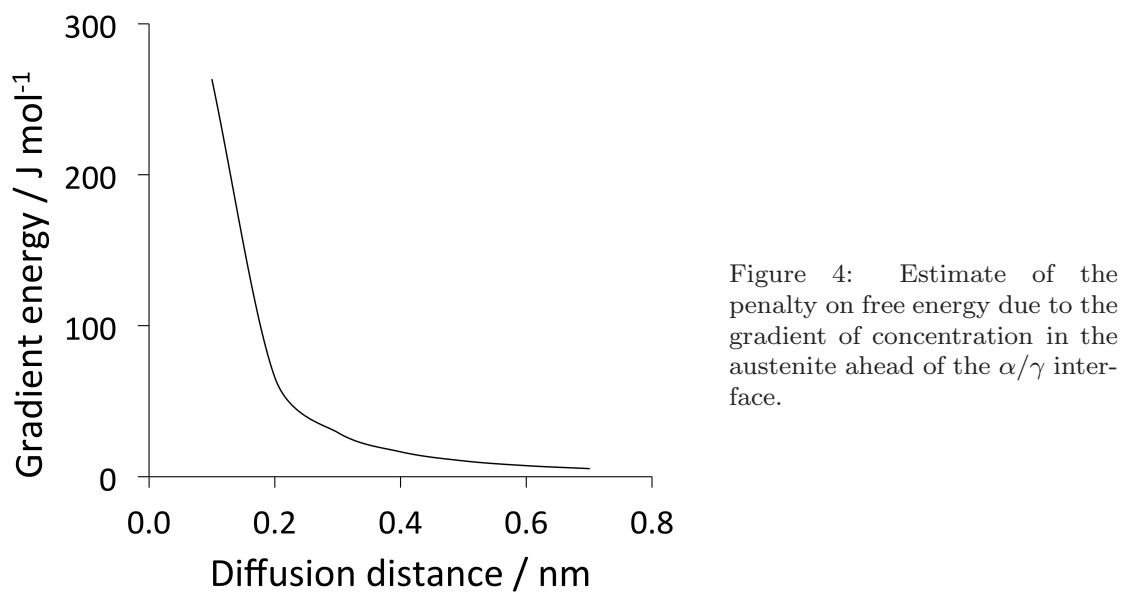

To put these calculations into perspective, equation 3 was applied to the growth rate calculations of Zhang et al. [24], who also conducted experimental measurements over the temperature range where a transition from P-LE to NP$\mathrm{LE}$ is expected. Using a value for the manganese diffusion coefficient $(1.05 \times$ $\left.10^{-5} \exp (-286000 / R T) \mathrm{m}^{2} \mathrm{~s}^{-1}, \quad R=8.3143 \mathrm{~J} \mathrm{~K}^{-1}\right)$ from [25], the parabolic rate constant $\left(5.17 \times 10^{-7} \mathrm{~m} \mathrm{~s}^{-0.5}\right)$ from Zhang et al. for $775^{\circ} \mathrm{C}$, the diffusion distance is estimated using equation 3 to be just $0.03 \mathrm{~nm}$. This not only is physically unrealistic but if it existed would have an intolerable penalty from the gradient energy term.

This basically means that the NP-LE calculations as implemented currently are wrong because they do not account for the gradient energy term. If such a term is incorporated then the concentration spike would be moderated to larger widths, making the extent of partitioning greater and the growth rate slower. This is precisely what happens during spinodal decomposition, where there is a wavenumber that receives maximum amplification [Fig. 4 20], so the actual wavelength observed is of the order of $10-20 \mathrm{~nm}$ [20].

\section{Conclusions}

1. If the constraint of local equilibrium at the $\alpha / \gamma$ interface is retained, then it is necessary to account for gradient energy terms in dealing with sharp concentration profiles. The penalty due to gradient energy will stop concentration profiles from becoming unrealistically narrow - judging from work on spinodal decomposition, a diffusion distance of the order 10$20 \mathrm{~nm}$ should be minimum although the actual number will depend on the magnitude of the free energy change available for transformation.

2. Another consequence of broader concentration profiles is that the extent of partitioning of solute between the austenite and ferrite may not then 
be 'negligible'.

3. Many solute-drag models rely on free energy being dissipated during diffusion within the interface. Since interfaces are thin, any concentration profiles must be narrow and may require an accounting for gradient energy terms.

4. A model in which growth with local equilibrium at the interface gives way to paraequilibrium when the concentration spike at the $\alpha / \gamma$ interface becomes unphysical is not viable, because narrow spikes will simply not develop.

5. The concept of paraequilibrium, where the ratio of substitutional solute to iron atoms $(\mathrm{X} / \mathrm{Fe})$ remains constant throughout transformation, may need to be looked at for the case of reconstructive transformations. Paraequilibrium transformation is inconsistent with the fact that reconstructive diffusion $[4,26]$ is essential in order to avoid any shape deformation involving shear components. ${ }^{7}$ It is worth emphasising that there is no experimental evidence to support a constant $(\mathrm{X} / \mathrm{Fe})$ ratio during any stage of allotriomorphic ferrite formation. It is likely that the concept of paraequilibrium is valid only for displacive transformations such as the formation of Widmanstätten ferrite in alloy steels, or the low-temperature precipitation of cementite [28].

Whereas we have identified that the gradient energy penalty can be a large portion of the driving force for transformation, the term now needs to be included in the kinetic theory. Thus, the flux from the interface will depend not just on the concentration gradient but on various derivatives of the gradient [equation 42.11 5]. This is work in progress, but it is worth pointing out that phase field models may not be useful in this context because of issues with interfacial widths [1]. Transmission electron microscopy indicates sharp $\alpha / \gamma$ interfaces with vivid dislocation structures [29]. The interfacial width is essentially a fitting or convenience parameter in phase field method.

Sharp interfaces have also been observed between the components of pearlite and the parent austenite [30]. Pearlite forms by a reconstructive transformation mechanism and always involves the partitioning of solutes irrespective of transformation temperature [31-33].

\section{References}

[1] R. S. Qin, H. K. D. H. Bhadeshia, Materials Science and Technology 26 (2010) 803-811.

\footnotetext{
${ }^{7}$ The need for reconstructive diffusion is made obvious by examining pure iron which can transform into ferrite without inducing a shape deformation, or into martensite in which case there is a large shape deformation involving a shear $[4,27]$. The reconstructive flow is necessary to allow reconstructive transformations to avoid the strain energy associated with displacive transformations.
} 
[2] M. Gouné, F. Danoix, J. Ågren, Y. Bréchet, C. R. Hutchinson, M. Militzer, H. Zurob, Materials Science \& Engineering R 92 (2015) 1-38.

[3] M. Hillert, in: Mechanism of Phase Transformations in Crystalline Solids, Monograph and Report Series No. 33, Institute of Metals, London, U.K., 1970, pp. 231-247.

[4] H. K. D. H. Bhadeshia, Progress in Materials Science 29 (1985) 321-386.

[5] J. W. Christian, Theory of Transformations in Metals and Alloys, Part I, Pergamon Press, Oxford, U. K., 3 edition, 2003.

[6] D. E. Coates, Metallurgical Transactions 3 (1972) 1203-1212.

[7] D. E. Coates, Metallurgical Transactions 4 (1973) 1077-1086.

[8] D. E. Coates, Metallurgical Transactions 4 (1973) 395-396.

[9] M. Hillert, Paraequilibrium, Technical Report, Swedish Institute for Metals Research, Stockholm, Sweden, 1953.

[10] A. Hultgren, Jernkontorets Annaler 135 (1951) 403-494.

[11] M. Hillert, Jernkontorets Annaler 136 (1952) 25-37.

[12] E. Rudberg, Jernkontorets Annaler 136 (1952) 91-112.

[13] H. I. Aaronson, H. A. Domian, TMS-AIME 236 (1966) 781-796.

[14] M. Hillert, A theory of nucleation of solid metallic solutions, Ph.D. thesis, Masschusetts Institute of Technology, Boston, USA, 1956.

[15] J. W. Cahn, J. E. Hilliard, Journal of Chemical Physics 28 (1958) 258-267.

[16] J. W. Cahn, J. E. Hilliard, Journal of Chemical Physics 31 (1959) 688-699.

[17] M. Hillert, Acta Metallurgica 9 (1961) 525-535.

[18] J. W. Cahn, Acta Metallurgica 9 (1961) 795-801.

[19] J. W. Cahn, Trans. Metall. Soc. AIME 242 (1968) 166-179.

[20] J. E. Hilliard, in: V. F. Zackay, H. I. Aaronson (Eds.), Phase Transformations, ASM International, Metals Park, Ohio, USA, 1970, pp. 497-560.

[21] H. E. Cook, D. de Fontaine, Acta Metallurgica 17 (1969) 915-924.

[22] T. Ujihara, K. Osamura, Acta Materialia 48 (2000) 1629-1637.

[23] T. Ujihara, K. Osamura, Materials Science \& Engineering A 312 (2001) $128-135$

[24] G. Zhang, R. Wei, M. Enomoto, D. W. Suh, Metallurgical \& Materials Transactions A 43 (2012) DOI: 10.1007/s11661-011-1000-9. 
[25] J. Fridberg, L.-E. Torndähl, M. Hillert, Jernkontorets Annaler 153 (1969) 263-276.

[26] H. K. D. H. Bhadeshia, Journal de Physique: Colloque C4 43 (1982) 449454.

[27] R. P. Zerwech, C. M. Wayman, Acta Metallurgica 13 (1965) 99-107.

[28] S. S. Babu, H. K. D. H. Bhadeshia, Materials Science and Technology 6 (1990) 1005-1020.

[29] F. A. Khalid, D. V. Edmonds, Acta Metallurgica et Materalia 41 (1993) 3421-3434.

[30] S. A. Hackney, G. J. Shiflet, Acta Materialia 35 (1987) 1007-1017.

[31] J. Chance, N. Ridley, Metallurgical Transactions A 12A (1981) 1205-1213.

[32] H. K. D. H. Bhadeshia, J. W. Christian, Metallurgical \& Materials Transactions A 21A (1990) 767-797.

[33] H. K. D. H. Bhadeshia, Bainite in steels: theory and practice, Maney Publishing, Leeds, U.K., 3rd edition, 2015. 